\title{
Catechol-O-methyltransferase Val I 58Met genotype in healthy and personality disorder individuals: Preliminary results from an examination of cognitive tests hypothetically differentially sensitive to dopamine functions
}

\author{
Winnie W Leung' \\ Margaret $M$ McClure' \\ Larry J Siever ${ }^{1,2}$ \\ Deanna M Barch ${ }^{3}$ \\ Philip D Harvey ${ }^{1,2}$ \\ 'Department of Veterans Affairs, \\ VISN 3 Mental Illness Research, \\ Education, and Clinical Center \\ (MIRECC), Bronx, NY, USA; \\ ${ }^{2}$ Department of Psychiatry, Mt. Sinai \\ School of Medicine, New York, NY, \\ USA; ${ }^{3}$ Departments of Psychology \\ and Psychiatry, Washington University, \\ St. Louis, MO, USA
}

\begin{abstract}
A functional polymorphism of the gene coding for Catechol-O-methyltrasferase (COMT), an enzyme responsible for the degradation of the catecholamine dopamine (DA), epinephrine, and norepinephrine, is associated with cognitive deficits. However, previous studies have not examined the effects of COMT on context processing, as measured by the AX-CPT, a task hypothesized to be maximally relevant to DA function. 32 individuals who were either healthy, with schizotypal personality disorder, or non-cluster A, personality disorder (OPD) were genotyped at the COMT Val158Met locus. Met/Met $(n=6), \mathrm{Val} / \mathrm{Met}(\mathrm{n}=10), \mathrm{Val} / \mathrm{Val}$ $(\mathrm{n}=16)$ individuals were administered a neuropsychological battery, including the AX-CPT and the N-back working memory test. For the AX-CPT, Met/Met demonstrated more AY errors (reflecting good maintenance of context) than the other genotypes, who showed equivalent error rates. Val/Val demonstrated disproportionately greater deterioration with increased task difficulty from 0-back to 1-back working memory demands as compared to Met/Met, while Val/Met did not differ from either genotypes. No differences were found on processing speed or verbal working memory. Both context processing and working memory appear related to COMT genotype and the AX-CPT and N-back may be most sensitive to the effects of COMT variation.
\end{abstract}

Keywords: COMT, dopamine, context processing, working memory, schizotypal personality disorder

Catecholamine neurotransmitter activity in the prefrontal cortex (PFC) exerts an influence over a range of cognitive functions. Specifically, catechol-O-methyltransferase (COMT) is an important enzyme involved in the regulation of catecholamines, including dopamine (DA). A single nucleotide polymorphism of the gene coding for COMT is associated with performance with working memory (Goldberg et al 2003) and executive functions in schizophrenia (Egan et al 2001; Joober et al 2002), with Val allele associated with increased enzymatic activity (leading to more catabolism of DA) and resultant poorer cognitive performance. In a recent study, Minzenberg et al (2006) demonstrated similar effects of the COMT genotype on cognitive performance in patients with schizotypal personality disorder (SPD). It is of interest that patients with SPD have been shown previously to manifest substantial abnormalities on tasks highly dependent on the functioning of the prefrontal cortex (PFC), including the ability to maintain contextual information in short term memory (Barch et al 2004). 
Context processing, defined as information actively maintained in such a form that it can be used to mediate later last-appropriate behavior, is particularly relevant in two ways. First, it has been suggested that a specific deficit in the ability to represent and maintain context information may help to explain deficits in working memory as well as other cognitive domains in schizophrenia and the spectrum disorders (Cohen and Servan-Schreiber 1992; Cohen et al 1999). In a neuroimaging study (Barch et al 2001), working memory deficits in patients with schizophrenia was shown to reflect impairment in context processing associated with a selective disturbance in dorsolateral PFC functions.

Second, context processing has been directly linked to DA function, in that D-amphetamine (D-AMP), a DA agonist, results in improved context processing (Servan-Schreiber et al 1998). Recent evidence from a double-blind, placebocontrolled trial of guanfacine treatment suggest that not only do patients with SPD perform more poorly on a context processing task than healthy control and patients with non-schizotypal personality disorders, pharmacological compounds increasing catecholamine activity in PFC exert a normalizing influence on context processing in SPD patients (McClure et al in press). Given its dopaminergic relevance, impaired context processing may also be related to the effects of the COMT genotype. However, there are no published data available on the relationship between COMT and context processing to date.

Tasks that measures executive functioning, such as the Wisconsin Card Sorting Test (WCST) (Egan et al 2001; Joober et al 2002; Malhotra et al 2002; Minzenberg et al 2006) and working memory, such as the N-back test (Goldberg et al 2003), and, by inference, the functioning of the dorsolateral PFC, have been shown to be related to the COMT genotype in healthy and schizophrenic individuals in some studies. Interestingly, Mattay et al (2003) reported better performance in carriers of the Val allele and a decline in the Met/Met group in a working memory task after a pharmacological challenge with amphetamine. The decline in performance in the Met/Met group after amphetamine intake suggests that the association between performance and DA levels has an inverted "U" shape characteristic. That is, activation of the DA system by working memory load and amphetamine pushes these subjects beyond their optimal activation level. However, other investigators did not find support that these tasks, presumed to be dopaminergic-depended tasks, were associated to COMT genotype (Tsai et al 2003; Ho et al 2005; Minzenberg et al 2006). Specifically, performance on measures like the WCST, digit span backward subtest of the WAIS-III, N-back test, and Trail Making (Ho et al 2005) were not associated with COMT genotype in healthy individuals or patients with schizophrenia. Additionally, COMT genotype did not exert an effect on tasks measuring verbal or visual delayed memory in healthy and schizotypal personality disorder individuals (Minzenberg et al 2006).

The AX Continuous Performance Test (AX-CPT) (Barch et al 2004) is a task specifically designed to assess context processing. During the AX-CPT, participants are presented with cue-probe pairs and are told to respond to an "X" (probe), but only when it follows an "A" (cue). The task also includes three types of non-target trials that allow one to selectively assess context processing deficits: AY trials ("A" cue followed by any letter other than "X"); BX trials (non-"A" cue followed by an "X" probe); and BY trials (non-"A" cue followed by a non-"X" probe). AX trials occur with high frequency (70\%), creating two important response biases. First, this high AX frequency creates a bias to make a target response to any stimulus following an " $A$ " cue (as a target " $\mathrm{X}$ " occurrence is highly likely following an "A" cue). In healthy individuals, maintenance of context is demonstrated by the tendency to make a false alarm response after occurrence of the "A" cue when not followed by an " $X$ " (leading to increased AY errors). Conversely, low levels of AY errors suggest reduced tendencies toward development of context representations. The second bias created by the high AX frequency is the tendency to make a target response to the "X" probe, as this is the correct response the majority of the time. On BX trials, maintenance of the context provided by the cue (non-A) reduces BX false alarms. Thus, on the AX-CPT, deficits in context processing are not indicated by an overall increase in false alarms, but rather a specific pattern of errors (decreased AY and increased BX).

In light of the current mixed findings regarding the association between DA-dependent tasks and the effects of COMT genotype, it is unclear whether COMT exerts a general effect on poor neurocognitive functioning or a specific deficit in one cognitive domain. Given the direct link to DA function and the PFC, the current study sought to examine whether COMT genotype variation has a specific impact on context processing. The Modified AX-CPT is the prototypical context processing task and is hypothetically one of the cognitive domains that is most DA relevant, and we hypothesized that it may be the most relevant task that is sensitive to the COMT effects, as compared to other DA-related tasks, including the N-back, measuring working memory, Trail Making, measuring processing speed and attention, and the Paced Auditory Serial Addition Test (PASAT) (Gronwall 1977), measuring maintenance and manipulation processes in 
verbal working memory. Previous studies have demonstrated that a functional genetic polymorphism of COMT influences prefrontal cognition in healthy individuals (Bruder et al 2005; Egan et al 2001; Malhotra et al 2002), healthy siblings of patients with schizophrenia spectrum disorders (Rosa et al 2004), and patients with schizophrenia (Egan et al 2001). Further, one published study reported that poorer performance on prefrontal-dependent tasks is associated with the $\mathrm{Val} / \mathrm{Val}$ genotype regardless of diagnosis in a group of healthy individuals and patients with SPD and OPD (Minzenberg et al 2006). Thus, in our ongoing study, we examined the shared effects of COMT variation on cognition in individuals with and without schizophrenia spectrum disorders, with the focus of examining tests hypothesized to be most sensitive to dopaminergic functions. As such, genotyping was collapsed across healthy individuals, patients with SPD, and patients with other, non-cluster A personality disorders (OPDs).

We predicted that subjects with the Val allele would show impairment in context processing, as evidence by a greater number of BX errors and a smaller number of AY errors on the AX-CPT, while subjects in the Met/Met group would demonstrate an inverse response pattern with a greater number AY errors and a smaller number of BX errors, reflecting intact context processing. We also predicted that, compared to the Met/Met group, subjects with the Val allele would show impaired working memory as measured by the N-back. In addition to evaluating N-back accuracy score at each condition with different levels of difficulty, we propose that examining the degree of improvement that takes place from one condition to the following condition with increased difficulty would allow us to better understand working memory deficit as it relates to COMT. We predicted that subjects with the Val allele would perform disproportionately worse as the task increases demands of working memory in comparison to the Met/Met group. Further, we predict that group differences in AX-CPT performance would yield a greater effect size, as compared to the N-back, suggesting that the AX-CPT is a more sensitive test to detect the effects on DA exerted by COMT.

\section{Methods}

\section{Participants}

As part of a larger study examining context processing in schizotypal personality disorder, 11 individuals with Diagnostic and Statistical Manual of Mental Disorders, 4th Edition (DSM-IV; American Psychiatric Association, 1994) SPD, two individuals with other, non-cluster A, DSM-IV personality disorders (OPD) and 19 healthy controls (HCs) were genotyped and evaluated on context processing and working memory. Participants with SPD and OPD were ascertained either through recruitment from the outpatient clinics at the Mount Sinai Medical Center and Bronx Veteran Affairs Medical Center, by advertisements in local newspapers, or by referral from psychiatrists and psychologists in the local community. The HCs were recruited from the local community through newspaper advertisements. Participants were excluded for (a) meeting criteria for current (within six months of testing) substance abuse or dependence, (b) a positive urine toxicology screen, (c) a lifetime diagnosis of a psychotic disorder or bipolar I disorder and (d) significant head trauma. Participants were assessed for Axis I psychopathology using the Structured Clinical Interview for DSM-IV (SCID; First et al 1995), by a master's level or doctoral level interviewer who did not know the participants' cognitive task performance. In addition, participants were assessed for Axis II pathology using the Structured Interview for the DSM-IV Personality Disorders (SIDP; Pfohl et al 1995). Consensus diagnoses were reached in a meeting of all raters with an expert diagnostician. OPD individuals were excluded from the current analyses if they met criteria for any Cluster A personality disorder (ie, paranoid personality disorder, schizoid personality disorder or if they met more than two criteria for SPD. Healthy controls were excluded if they had either a personal or family history of a major Axis I disorder (eg, schizophrenia), or a personal history of an Axis II disorder. All participants signed informed consent forms in accordance with the approvals of Institutional Review Boards at each research site, where ethical approval for the study procedures was obtained.

\section{Tasks and apparatus AX-CPT tasks}

Participants performed the modified version of the AX-CPT, in which sequences of letters were visually presented one at a time in a continuous fashion on a computer display. Subjects were instructed to identify target and non-target trials with a button press using separate fingers on the same hand. Target trials were defined as a cue-probe sequence, in which the letter " $A$ " appeared as the cue, and the letter " $X$ " appeared as the probe. The remaining letters of the alphabet served as invalid cues (ie, cues that were not A's) and non-target probes (ie, probes that were not X's), with the exception of the letters $\mathrm{K}$ and $\mathrm{Y}$, which were excluded due to their similarity in appearance to the letter $\mathrm{X}$. Letter sequences were presented in pseudorandom order, such that target $(\mathrm{AX})$ trials occurred with $70 \%$ frequency, and non-target trials occurred with $30 \%$ frequency. Non-targets were divided evenly $(10 \%$ each) among the following trial types: BX trials, in which 
an invalid cue (ie, non-A) preceded the target; AY trials, in which a valid cue was followed by a non-target probe (ie, non-X); and BY trials, in which an invalid cue was followed by a non-target probe. The delay between cue and probe was manipulated so that half of the trials had a short delay and half had a long delay. On short delay trials, the cue-probe interval was $1 \mathrm{sec}$, and the inter-trial interval was $4900 \mathrm{msec}$. On long delay trials, the cue-probe interval was $5 \mathrm{sec}$ and the inter-trial interval was $1 \mathrm{sec}$. Thus, the total trial duration was equivalent across conditions, providing a means of controlling for general factors that might affect performance (eg, pace of the task, response frequency, total time on task). The task was presented in 4 blocks of 50 trials, all of which were either short ( 2 blocks) or long ( 2 blocks) delay trials, with the order of short and long delay blocks counterbalanced across subjects.

Stimuli were presented centrally, for a duration of $300 \mathrm{msec}$, in 24-point uppercase Helvetica font. Subjects were instructed to respond to both cue and probe stimuli, pressing one button for targets and another button for non-targets (cues were always considered non-targets). Responses were recorded on a specially constructed button box connected to the computer that recorded response choice and reaction time with $1 \mathrm{msec}$ accuracy. For right-handed individuals, responses were made with the middle (nontarget, middle button) and index (target, right button) fingers of the right hand. For left-handed individuals, responses were made with the middle (non-target, middle button) and index (target, right button) fingers of the left hand. Following previous work (Barch et al 2004), subjects were allowed a total of $1300 \mathrm{msec}$ from stimulus onset in which to respond. Responses slower than this limit were not recorded, and elicited feedback (a "bloop" sound) as a prompt to increase speed. The task was run on Apple Macintosh computers, using PsyScope software for stimulus presentation and data collection (Cohen et al 1993).

\section{$\mathrm{N}$-back working memory task}

The N-back is a commonly used measure of working memory (Braver et al 1997; Casey et al 1995; Cohen et al 1996) that has been frequently shown to elicit performance deficits among individuals with schizophrenia and their unaffected relatives (Callicott et al 2000; Egan et al 2001; Menon et al 2001; Perlstein et al 2001; Barch et al 2002; Callicott et al 2003). The N-back test manipulates complexity with working memory load, rather than retention duration or interference conditions. Materials for the N-back task were similar to those used by Braver et al (1997). In the current study, participants observed letters presented on a computer screen one at a time. There were three conditions: (1) 0-back, (2) 1-back, and (3) 2-back. In the 0-back condition, participants responded to a single pre-specified target letter (eg, X). In the 1-back condition, the target was any letter identical to the one immediately preceding it (ie, one trial back). In the 2-back condition, the target was any letter identical to the one presented 2 trials back. Thus, working memory load is increased incrementally from the 0-back to the 2-back conditions.

Stimuli were presented as single letters appearing centrally in 24-point Helvetica font, white against a black background, subtending a visual angle of approximately 3 degrees. All consonants of the alphabet were used as stimuli with the exception of $\mathrm{L}$ (because it is easily confused with the number " 1 ") and W (because it is the only two syllable letter of the alphabet). Vowels were excluded. Further, the case of the presented lettered-stimuli changed randomly throughout the trials. Stimuli were presented in a pseudorandom sequence of consonants, randomly varying in case in order to prevent participants from relying on strategies of perceptual familiarity for responding. Stimuli were presented centrally on a controlled computer display for $500 \mathrm{msec}$. The inter-stimulus interval (ISI) was $2500 \mathrm{msec}$. Targets were presented on $33 \%$ of the trials. Conditions were presented in blocks of 25 trials, with three blocks at each load level (0-, 1-, 2-back) presented in a counterbalanced order. The ISI and target density for the N-back test were selected in order to be consistent with prior studies using this test (Casey et al 1995; Cohen et al 1996; Braver et al 1997).

\section{WAIS-III vocabulary and block design}

The Vocabulary and Block Design subtests of the WAIS-III (Wechsler, 1997) are the best correlates of overall IQ (Tulsky et al 1997; Wechsler 1997). These subtests were administered to obtain an estimate of general level of intellectual ability.

\section{Other neuropsychological measures}

Trail Making Part A and B (Reitan and Wolfson, 1993) is a timed test that measures processing and psychomotor speed. On part A, subjects are presented with numbers from one to 25 randomly placed on a sheet of paper and instructed to connect the numbers in their correct numerical order as quickly as possible. Part B is analogous to Part A with the addition of letters and subjects are instructed to alternate between numbers and letters in numerical and alphabetical order. The PASAT (Gronwall 1977) measures maintenance 
and manipulation processes in verbal working memory. On this test, subjects listen to a tape recorded voice presenting a series of numbers and are asked to add each adjacent pair of numbers and respond by verbalizing the sum. There are 50 trials at a rate of one digit per two seconds, with total correct detections as the dependent variable.

Participants were tested in a single testing session. Task order was counterbalanced across participants. Prior to performance of the first block of each computerized task, standardized instructions describing the task appeared on the computer, and the experimenter answered any remaining questions regarding them. Participants were asked to respond as quickly as possible to each stimulus while maintaining accuracy. One full block of trials was then performed as practice prior to administration of the experimental trials for that condition. This ensured that subjects understood the instructions and were performing the task appropriately.

\section{COMT genotyping}

Blood samples were collected from all participants and DNA was extracted. COMT Val108/158Met (rs4680) genotype was determined as a restriction fragment length polymorphism after polymerase chain reaction (PCR) amplification from genomic DNA. DNA sequences were obtained from GenBank (accession no: Z26491). The sequence of primers and probes were as follows: forward primer, 5'-TCGAGATCAACCCCGACTGT-3'; reserve primer, 5'-AACGGGTCAGGCATGCA-3'; probe for Val allele, 5'-6FAM-CCTTGTCCTTCACGCCAGCGA-3'-TAMRA; and probe for Met allele, 5'-VIC-ACCTTGTCCTTCATGCCAGCGA-3'-TAMRA. The genotyping protocol has been described elsewhere (Xu et al 2002; Minzenberg et al 2006). Briefly, individual genotyping was measured by using Applied Biosystems (ABI; Foster City, California, USA) 7900 Sequence Detector with a $5 \mu$ reaction volume and 1-3 mg DNA. Primer concentration was $900 \mathrm{nmol} / \mathrm{l}$ for each primer and $100 \mathrm{nmol} / 1$ concentration for each probe. Annealing temperature was $63.5^{\circ} \mathrm{C}$ with 35 cycles. Genotyping was clustered by using SDS 2.2 software (ABI). Each cluster represented homozygous Val allele, heterozygous Val/Met, Homozygous Met/Met allele and no DNA template control.

\section{Data analysis}

Participants were collapsed diagnostic groups for genotyping and analyses were conducted with group comparison between the different COMT genotypes for two reasons. First, polymorphism of COMT also influences prefrontal cognition in healthy individuals as well as schizophrenia patients, and performance on prefrontaldependent tasks is associated with the Val/Val genotype regardless of diagnosis. Second, the focus of the paper is to investigate the association between COMT and a task (ie, AX-CPT) hypothesized to be maximally relevant to DA function. For the AX-CPT, analyses focused on error rates for the two error types most related to effective context processing, BX and AY, for both the short and long delay intervals. Context processing data were analyzed using analyses of variance (ANOVAs) with genotype as a between-subject factor and trial type and delay as within-subject factors. For the N-back, accuracy scores were calculated for each participant across the three conditions (0-back, 1-back, 2-back). ANOVAs were conducted to examine the accuracy responses from the N-back with genotype as a between-subject factor and condition as a within-subject factor. Additionally, change scores were calculated to capture changes in performance as the $\mathrm{N}$-back test increased in difficulty from 0 to 1 -back and 1 to 2 -back. While accuracy scores provide information on performance level at each task condition, change scores would yield additional information regarding the proportion of worsening as load increased in the task Greater changes indicate greater vulnerability to increases in processing demands. A series of Univariate ANOVAs were conducted to examine changes in performance with genotype as the between-subject factor and change scores as the dependent variable. For all posthoc analyses, planned contrasts were conducted for multiple pairwise comparisons and Bonferroni was used to adjust for the alpha level.

\section{Results \\ Demographic information}

Three groups of COMT genotypes were identified, including Met/Met $(n=6), \mathrm{Val} / \mathrm{Met}(\mathrm{n}=10)$ and $\mathrm{Val} / \mathrm{Val}(\mathrm{n}=16)$. Demographic characteristics of the genotype groups are shown in Table 1. One participant has missing demographic data. The genotypes did not differ on age, $F(2,28)=0.60$, $p=0.56$, education, $F(2,28)=0.56, p=0.58$, ethnicity, $\chi^{2}(8)=7.55, p=0.48$, gender composition, $\chi^{2}(2)=0.02$, $p=0.99$, or estimated IQ as measured by WAIS Vocabulary, $F(2,28)=0.65, p=0.53$ or Block Design, $F(2,27)=1.20$, $p=0.32$. The majority of the participants $(84.4 \%)$ were righthanded individuals, but again, the genotypes did not differ on handedness, $\chi^{2}(2)=1.40, p=0.50$. 
Table I Sample characteristics

\begin{tabular}{|c|c|c|c|c|c|c|}
\hline & \multicolumn{6}{|c|}{ Group } \\
\hline & \multicolumn{2}{|c|}{ Met/Met } & \multicolumn{2}{|c|}{ Val/Met } & \multicolumn{2}{|c|}{$\mathrm{Val} / \mathrm{Val}$} \\
\hline & $\mathbf{M}$ & SD & $\bar{M}$ & SD & $M$ & SD \\
\hline Age (in years) & 31.7 & 10.7 & 31.0 & 11.4 & 35.8 & 10.8 \\
\hline Sex (\% male) & 66.7 & - & 70.0 & - & 68.8 & - \\
\hline Handedness (\% right) & 100 & - & 88.9 & - & 81.3 & - \\
\hline Education (in years) & 16.1 & 2.6 & 16.7 & 4.1 & 15.1 & 3.7 \\
\hline Vocabulary scores & 9.8 & 2.4 & 10.2 & 2.7 & 11.13 & 2.6 \\
\hline Block design scores & 12.0 & 3.0 & 12.7 & 3.7 & 10.6 & 3.1 \\
\hline
\end{tabular}

\section{Effects of COMT genotype on context processing}

For the purposes of this study, we will focus on main effects of genotype or interaction with genotype in all of the analyses presented below, as our hypotheses focused on genotypic differences. The error data from the AX-CPT was analyzed using a 3-factor ANOVA, with genotype (Met/Met, Val/Met, $\mathrm{Val} / \mathrm{Val}$ ) as a between-subject factor, and both delay (short, long) and error type (AX, AY, BX, BY) as within-subject factors. Descriptive statistics for the AX-CPT are presented in Table 2. The ANOVA did not reveal a significant main effect of genotype, $F(2,29)=0.54, p=0.59$. However, there was a non-significant trend for an error type by genotype interaction, $F(6,56)=2.06, p=0.07$, with the $\mathrm{Val} / \mathrm{Val}$ group making more BX than AY errors, while the Val/Met and Met/Met groups made more AY and BX errors. Given our small sample sizes, we followed up the interaction although it was nonsignificant.

Follow up univariate ANOVAs showed a significant genotype difference in AY errors during the short delay condition, $F(2,29)=3.77, p<0.05, R^{2}=0.21$. Compared to Met/Met, Val/Met $(p<0.05)$ and Val/Val $(p<0.05)$ made

Table 2 Standard AX-CPT errors

\begin{tabular}{|c|c|c|c|}
\hline \multirow[b]{3}{*}{ Error type } & \multicolumn{3}{|l|}{ Group } \\
\hline & Met/Met & Val/Met & Val/Val \\
\hline & $\overline{M(S D)}$ & $M(S D)$ & $M(S D)$ \\
\hline \multicolumn{4}{|l|}{ Short delay } \\
\hline$A X$ & $3.8(6.5)$ & I.4(I.5) & $1.6(3.3)$ \\
\hline AY & $20.0(26.8)$ & $4.0(7.0)$ & $4.4(6.4)$ \\
\hline$B X$ & $3.3(5.2)$ & $5.3(7.7)$ & $7.5(25.2)$ \\
\hline BY & I.7 (4.I) & $2.3(4.8)$ & $2.0(4.4$ \\
\hline \multicolumn{4}{|l|}{ Long delay } \\
\hline$A X$ & 20.1 (25.9) & $7.6(11.6)$ & I I.8 (20.0) \\
\hline AY & $16.7(24.2)$ & $11.2(11.2)$ & $1.9(5.4)$ \\
\hline$B X$ & $3.5(5.5)$ & II.4(I5.5) & I $3.3(25.5)$ \\
\hline BY & $0.0(0.0)$ & $0.0(0.0)$ & I.4 (5.6) \\
\hline
\end{tabular}

Note: Error data are proportions of errors. significantly fewer AY errors, but Val/Met and Val/Val did not differ. Similarly, there was also a significant genotype difference in $\mathrm{AY}$ errors during the long delay $F(2,29)=$ 3.69), $p<0.05, R^{2}=0.20$, with Met/Met making more $\mathrm{AY}$ errors compare to the $\mathrm{Val} / \mathrm{Val}(p<0.05)$ while the Val/Met did not differ from the other two groups $(p>0.05)$. There were no genotype differences in $\mathrm{BX}$ errors during the short $F(2,29)=0.12, p=0.89$, or long $F(2,29)=0.51, p=0.61$ delay. These results suggest that although the groups did not differ on BX false alarms, Met/Met subjects demonstrated better maintenance of context relative to Val/Met and Met/ Met subjects, as reflected by the AY errors.

\section{Effects of COMT genotype on working memory}

The accuracy data from the N-back were analyzed using a 2factor ANOVA with genotype (Met/Met, Val/Met, Val/Val) as a between subject factor and condition (0-back, 1-back, 2-back) as a within-subject factor. A significant main effect of condition was found, $F(2,28)=8.21, p<0.01$, but there was no significant main effect of genotype, $F(2,29)=0.61$, $p=0.55$, or interaction, $F(4,58)=1.74, p=0.15$. As displayed on Table 3, participants performed the worst at 2-back compared to the other two conditions ( $p$ 's $<0.01$ ), though there were no differences in performance between 0-back and 1-back $(p>0.05)$.

Genotype comparison analyses were conducted to examine changes in performance as the task increased in difficulty (ie, the difference in accuracy score from one condition to another). This mechanism would provide more meaning information on differential deterioration as the task becomes more demanding. Change scores were calculated to capture changes in performance from 0-back to 1-back, 1-back to 2-back, and 0-back to 2-back. The ANOVA revealed a significant genotype difference in performance from 0-back to 1 -back, $F(2,29)=3.66, p<0.05), R^{2}=0.20$. As shown in Figure 1, Val/Val subjects had a greater change score compared to Met/Met subjects $(p<0.05$ ), suggesting a greater

Table $3 \mathrm{~N}$-back working memory accuracy scores

\begin{tabular}{llll}
\hline & Group & & \\
\cline { 2 - 4 } & Met/Met & Val/Met & Val/Val \\
& M (SD) & M (SD) & M (SD) \\
\hline 0-back & $88.9(I I .0)$ & $94.7(5.8)$ & $97.9(I .6)$ \\
I-back & $92.5(8.6)$ & $92.7(5.9)$ & $92.8(6.4)$ \\
2-back & $86.4(5.2)$ & $88.0(12.1)$ & $86.7(9.6)$ \\
\hline
\end{tabular}

Note: Response data are proportion of correct responses. 


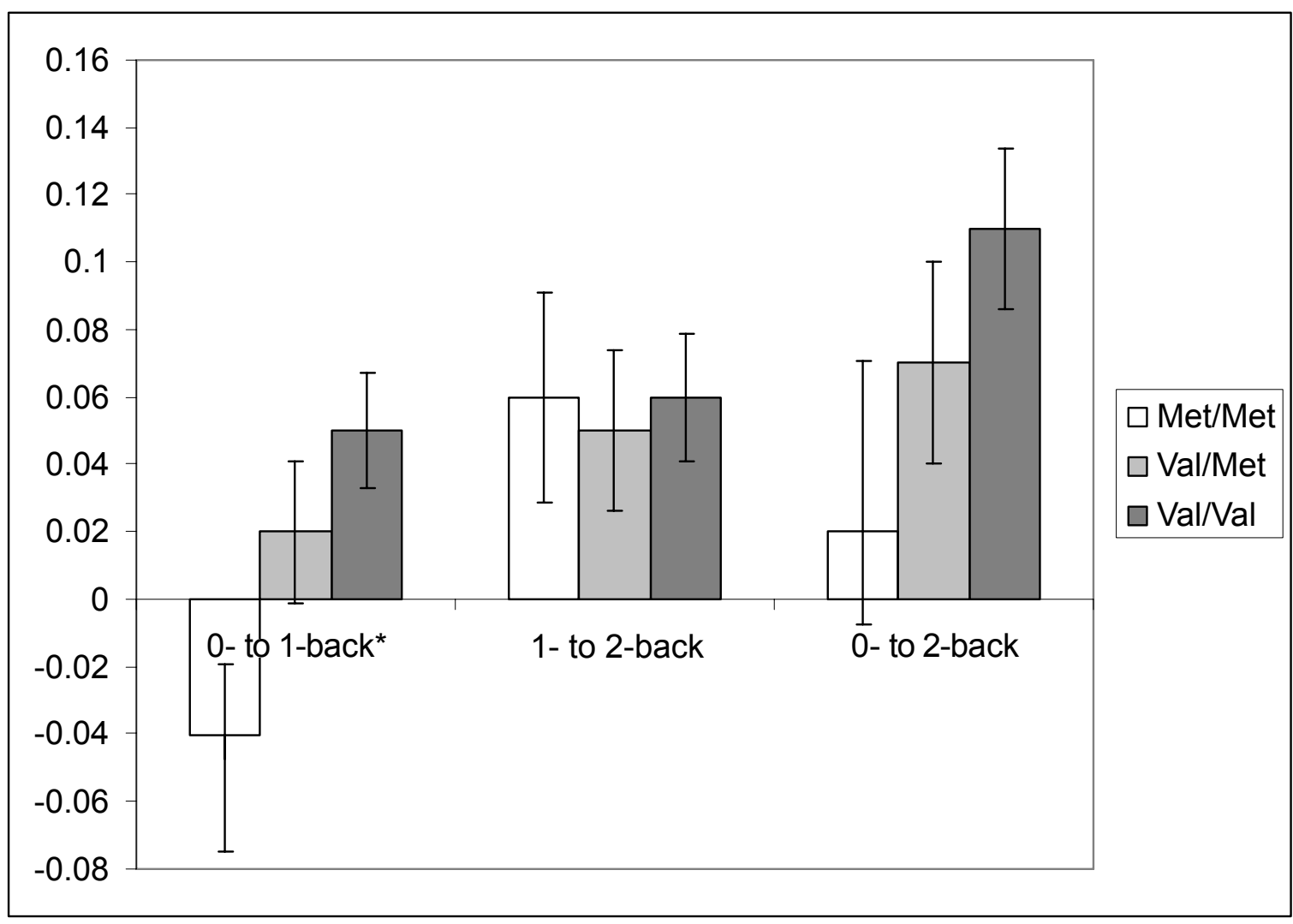

Figure I Proportions of change in performance on the n-back test as captured by difference scores from 0- to I-back, I- to 2-back and 0- to 2-back. Note: Larger change score indicates a greater decrease in accuracy as the condition increased in difficulty because performance is expected to be worse as the more demanding condition (ie, I-back) relative to the less demanding condition (ie, 0 -back). $* \mathrm{Val} / \mathrm{Val}$ subjects demonstrated a greater change score compared to Met/Met subjects, $\mathrm{P}<0.05$.

deterioration going from 0 - to 1-back, while $\mathrm{Val} / \mathrm{Met}$ subjects did not differ from the other groups. There were no significant genotype differences in change of performance from 1- to 2-back $F(2,29)=0.12, p=0.89$, or 0- to 2-back, $F(2,29)=$ $2.04, p=0.15$, although the $\mathrm{Val} / \mathrm{Val}$ group clearly showed the largest deterioration from 0 to 2 back.

\section{Effects of COMT genotype on other PFC indices}

A series of ANOVAs were conducted to examine genotype group differences on processing speed, as indexed by Trails Making A and B, and verbal working memory, as indexed by the PASAT. One subject from the Val/Met genotype had missing data on these measures and was not included in these analyses. The genotypes did not differ on Trail Making part A, $F(2,28)=3.32, p=0.51$, Trail Making part B, $F(2,28)=$ $2.24, p=0.13$, or the PASAT, $F(2,28)=0.29, p=0.75$.

\section{Discussion}

The goal of the current study was to examine the effects of COMT genotype on context processing, as well as to replicate previous findings of working memory deficit in carriers of the Val allele. We predicted that the Val/Met and $\mathrm{Val} / \mathrm{Val}$ genotypes would demonstrate impairment in context processing, and that this impairment would not be found in the Met/Met group.

In the modified AX-CPT context processing is best understood by the pattern of errors, rather than the overall number of errors, that an individual makes. Specifically, a greater number of AY errors suggest development of a response bias reflecting intact context processing and individuals with intact context processing tend to make a smaller number of BX errors. Impaired context processing, on the other hand, is indicated by the reverse pattern: greater numbers of $\mathrm{BX}$ and smaller numbers of $\mathrm{AY}$ errors. While we did not find a main effect of genotype, there was a non-significant trend for a genotype by condition interaction. This is likely due to the low statistical power as a result of the small sample size because additional post-hoc tests showed that, during both the short and long delays of the AX-CPT, participants in the Met/Met group demonstrated a greater number of AY errors, as compared to the other two 
groups. Further, COMT genotype accounted for 20\%-21\% of variance in AX-CPT performance which is substantial compared to previous results of shared variance with other cognitive tests (Egan et al 2001; Malhotra et al 2002). There were, however, no differences in the number of BX errors in either the short or long delay condition, which is interesting in the context of the recent results of McClure et al (2006). In that study we found that the beneficial effects of guanfacine, an adrenergic agonist, were greater on AY than BX errors. In a study examining executive functioning and COMT, Egan et al (2001) reported that COMT genotype accounted for $4 \%$ of the variance in frequency of preseverative errors on the WCST, while the current study found that COMT genotype accounted for about $20 \%$ of the variance on the AX-CPT. This finding is quite robust and is consistent with our hypothesis that the AX-CPT is dopaminergic task that is sensitive to the effects of COMT. In support of the idea that the small sample that we collected is still representative, the Met/Met group in our sample demonstrated an average of AY error rate of $18.3 \%$, which is similar, albeit slightly larger, to the rates reported by McDonald et al (2003) and Barch et al (2001), Thus, the larger variance accounted for statistics may truly reflect differences in task sensitivity to dopaminergic effects.

Although we did not find genotype differences in performance on the N-back working memory test when accuracy scores were analyzed at each condition of the test, $\mathrm{Val} / \mathrm{Val}$ subjects demonstrated a larger change score from 0-back to 1back, as compared to the Met/Met group. Since performance is expected to be worse at the more difficult condition (ie, 1-back) relative to the less demanding condition (ie, 0 -back), this larger change score demonstrated by the Val/Val indicates a greater decrease in accuracy from a less demanding condition to a more demanding condition. As such, the $\mathrm{Val} / \mathrm{Val}$ subjects performed disproportionately worse as they progressed from 0-back to 1-back compared to the Met/Met group. Therefore, findings of a larger change in performance 0 -back to 1-back in the $\mathrm{Val} / \mathrm{Val}$ group provides evidence of working memory deficits in those individuals, which is in line with previous findings (Goldberg et al 2003). We propose that this method of examining working memory measured by the N-back may be of particular utility in future studies. In contrast, there was no significant genotype difference for the change score from 0 -back to 2-back, suggesting that the difficulty level in the 2-back condition may be too high for all participants and thus resulted in reduced sensitivity.

We predicted that the AX-CPT would be a more sensitive task to detect effects of COMT in comparison to the
N-back working memory task. COMT genotype explained comparable amounts of variance in both tasks (20\%), but only with a different analytic plan than previously employed. Consistent with our hypothesis, processing speed and verbal working memory were not associated to the effects of COMT genotype.

A reformulation of the COMT genotype effect on cognition was recently provided by Bilder et al (2004), which suggested that the functional effects of the COMT polymorphism may be better understood from the perspective of the tonicphasic DA theory. Bilder et al (2004) hypothesized that the Met allele is associated with increased tonic and decreased phasic DA transmission, leading to increased stability but reduced flexibility of neural network activation states that are central to aspects of working memory. It was suggested that these effects may be beneficial or detrimental depending the phenotype and environmental demands (ie, cognitive task). Thus, given our findings, the AX-CPT and N-back may be DA-dependent tasks that require the stability of networks that underlies working memory, rather than the flexibility of neural programming.

One major limitation of the current study relates to the small sample size. However, it should be noted that large effect sizes were found despite the small number of subjects. Future studies with a larger sample are needed to replicate the current findings. Further, examination of diagnosis $\times$ genotype interactions would be of interest as well.

One interesting empirical question that the current findings point to is whether or not COMT genotype plays a significant role in functional disability among patients with schizophrenia and schizotypal personality disorder. There is a substantial amount of evidence that schizophrenia is marked by profound functional impairments, including occupational (Carpenter and Strauss 1991; McGurk and Meltzer 2000) and social functioning (Mueser et al 1991; Green 1996; Green et al 2000). Further, across multiple studies, cognitive impairment has been found to be consistently related to work outcomes (Bryson et al 1998; McGurk and Meltzer 2000; Suslow et al 2000; Tsang et al 2000; Mueser et al 2001) and cognitive functioning uniquely predicted functional outcomes in patients (Green 1996) and individuals at risk for schizophrenia (Niendam et al 2006) over and above clinical symptoms. Thus, it would be interesting to investigate whether COMT genotype mediates the relationship between cognitive deficits and functional disability in patients with schizophrenia and schizotypal personality disorder.

In summary, preliminary results of the current study provided some evidence that context processing and working 
memory are associated with COMT genotype variation, and the AX-CPT and N-back are two theoretically dopaminergic dependent tasks most sensitive in capturing the effects of COMT. Processing speed and verbal working memory were not found to be related to COMT genotype in the current sample. Taken together, this study provides evidence that variation in COMT genotype does not lead to general impairment of cognitive functioning, but that it uniquely affects two specific prefrontal domains, context processing and working memory.

\section{Acknowledgments}

This research was supported by NIMH Grant Number MH 56140 to Dr. Siever, NIMH Grant Number MH 63116 to Dr. Harvey, and by the VA VISN 3 MIRECC.

\section{References}

American Psychological Association, 1994. Diagnostic and statistical manual of mental disorders (4th ed) Washington, DC: Author.

Barch DM, Carter CS, Braver TS, et al. 2001. Selective deficits in prefrontal cortex function in medication-naïve patients with schizophrenia. Arch Gen Psychiatry, 58:280-8.

Barch DM, Csermansky J, Conturo T, et al. 2002. Working and long-term memory deficits in schizophrenia. Is there a common underlying prefrontal mechanism? J Abnorm Psychol, 111:478-94.

Barch DM, Mitropoulou V, Harvey PD, et al. 2004. Context processing deficits in schizotypal personality disorder. J Abnorm Psychol, 113:556-68.

Bilder RM, Volavka J, Lachman HM, et al. 2004. The CatecholO-Methyltransferase polymorphism: Relations to the tonicphasic dopamine hypothesis and neuropsychiatric phenotypes. Neuropsychopharmacology, 29:1943-61.

Braver TS, Barch DM, 2002. A theory of cognitive control, aging cognition, and neuromodulation. Neurosci Biovehav Rev, 26:809-17.

Braver TS, Cohen JD, Nystrom LE, et al. 1997. A parametric study of prefrontal cortex involvement in human working memory. Neuroimage, 5:49-62.

Bruder GE, Keilp JG, Xu H, et al. 2005. Catechol-O-methyltransferase (COMT) genotypes and working memory: Associations with differing cognitive operations. Biol Psychiatry, 58:901-7.

Bryson G, Bell MD, Kaplan E, et al. 1998. The functional consequences of memory impairments on initial work performance in people with schizophrenia. J Nerv Ment Dis, 186:610-5.

Callicott JH, Bertolino A, Mattay VS, et al. 2000. Physiological dysfunction of the dorsolateral prefrontal cortex in schizophrenia revisited. Cereb Cortex, 10:1078-92.

Callicott JH, Egan MF, Mattay VS, et al. 2003. Abnormal fMRI response of the dorsolateral prefrontal cortex in cognitive intact siblings of patients with schizophrenia. Am J Psychiatry, 160:709-19.

Carpenter WT, Strauss JS. 1991. The prediction of outcome in schizophrenia IV: eleven year follow-up of the Washington IPSS cohort. J Nerv Ment Dis, 179:517-25.

Casey BJ, Cohen JD, Jezzard P, et al. 1995. Activation of prefrontal cortex in children during a non-spatial working memory task with functional MRI. Neuroimage, 2:221-9.

Cohen JD, Barch DM, Carter C, et al. 1999. Context-processing deficits in schizophrenia: Converging evidence from three theoretically motivated cognitive tasks. J Abnorm Psychol, 108:120-33.

Cohen JD, Braver TS, Perlstein WM, et al. 1996. Temporal dynamics of cortical activity in verbal working memory. Neuroimage, 3:S537.
Cohen JD, MacWhinnery B, Flatt MR, et al. 1993. PsyScope: a new graphic interactive environment for designing psychology experiments. Behav Res Methods Instrum Comput, 25:257-71.

Cohen JD, Servan-Schreiber D. 1992. Context, cortex, and dopamine: A connectionist approach to behavior and biology in schizophrenia. Psychol Rev, 99:45-77.

Egan MF, Goldberg TE, Kolachana BS, et al. 2001. Effect of COMT Val108/158Met genotype on frontal lobe function and risk for schizophrenia. Proc Natl Acad Sci, 98:6917-22.

First MB, Spitzer RL, Gibbon M, et al. 1995. Structured clinical interview for DSM-IV Axis I disorders - patient edition (SCID-I/P, Version 2.0). New York: New York State Psychiatric Institute.

Green MF. 1996. What are the functional consequences of neurocognitive deficits in schizophrenia. Am J Psychiatry, 153:321-30.

Green MF, Kern RS, Braff DL, et al. 2000. Neurocognitive deficits and functional outcome in schizophrenia: are we measuring the "right stuff'?. Schizophr Bull, 26:119-36.

Goldberg TE, Egan MF, Gscheidle T, et al. 2003. Executive subprocesses in working memory. Relationship to catechol-O-methyltransferase Val158Met genotype and schizophrenia. Arch Gen Psychiatry, 60:889-96.

Gronwall DMA. 1977. Paced auditory serial-addition task: a measure of recovery from concussion. Percept Motor Skills, 44:367-73.

Ho BC, Wassink TH, O'Leary DS, et al. 2005. Catechol-O-methyl transferase Val158Met gene polymorphism in schizophrenia: working memory, frontal lobe MRI morphology and frontal cerebral blood flow. Mol Psychiatry, 10:287-98.

Joober R, Gauthier J, Lal S, et al. 2002. Catechol-O-methyltransferase Val-180/158-Met gene variants associated with performance on the Wisconsin Card Sorting Test. Arch Gen Psychiatry, 59:662-3.

MacDonald AW, Pogue-Geile MF, Johnson MK, et al. 2003. A specific deficit in context processing in the unaffected siblings of patients with schizophrenia. Arch Gen Psychiatry, 60:57-65.

Malhotra AK, Kestler LJ, Mazzanti C, et al. 2002. A functional polymorphism in the COMT gene and performance on a test of prefrontal cognition. Am J Psychiatry, 159:652-4.

Mattay VS, Goldberg TE, Fera F, et al. 2003. Catechol O-methyltransferase Val158Met genotype and individual variation in the brain response to amphetamine. Proc Natl Acad Sci, 100:6186-91.

McClure MM, Barch DM, Romero MJ, et al. 2006. in press. The effects of guanfacine on context processing abnormalities in schizotypal personality disorder. Biol Psychiatry, Epub ahead of print, published online 1 September 2006. URL: http://www.sciencedirect.com.

McGurk SR, Meltzer HY. 2000. The role of cognition in vocational functioning in schizophrenia. Schizophr Res, 45:175-84.

Menon V, Anagnoson RT, Mathalon DH, et al. 2001. Functional neuroanatomy of auditory working memory in schizophrenia: relation to positive and negative symptoms. Neuroimage, 13:433-46.

Minzenberg MJ, Xu K, Mitropoulou V, et al. 2006. Catechol-O-methyltransferase Val158Met genotype variation is associated with prefrontaldependent task performance in schizotypal personality disorder patients and comparison groups. Psychiatr Genet, 16:117-24.

Mueser KT, Bellack AS, Douglas MS, et al. 1991. Prevalence and stability of social skill deficits in schizophrenia. Schizophr Res, 5:167-76.

Mueser KT, Salyers MP, Mueser PR. 2001. A prospective analysis of work in schizophrenia. Schizophr Bull, 27:281-96.

Niendam TA, Bearden CE, Johnson JK, et al. 2006. Neurocognitive performance and functional disability in the psychosis prodrome. Schizophr Res, 84:100-11.

Perlstein WH, Carter CS, Noll DC, et al. 2001. Relation of prefrontal cortex dysfunction to working memory and symptoms in schizophrenia. Am J Psychiatry, 158:1105-13.

Pfol B, Blum N, Zimmerman M. 1995. Structured Interview for DSM-IV Personality (SCIP-IV). Iowa City: University of Iowa, College of Medicine.

Reitan RM, Wolfson D. 1993. The Halstead-Reitan Neuropsychological Test Battery: Theory and clinical interpretation (2nd ed) Tucson, AZ: Neuropsychological Press. 
Rosa A, Peralta V, Cuesta MJ, et al. 2004. New evidence of association between COMT gene and prefrontal neurocognitive function in healthy individuals from sibling pairs discordant for psychosis. Am JPsychiatry, 161:1110-2.

Servan-Schreiber D, Carter CS, Bruno RM, et al. 1998. Dopamine and the mechanisms of cognition: Part II. D-Amphetamine effects in human subjects performing a selective attention task. Biol Psychiatry, 43:723-9.

Suslow T, Schonauer K, Ohrmann P, et al. 2000. Prediction of work performance by clinical symptoms and cognitive skills in schizophrenic outpatients. J Nerv Ment Dis, 188:116-8.

Tsai SJ, Yu YW, Chen JT, et al. 2003. Association study of a functional catecho-O-methyltransferase-gene polymorphism and cognitive function in healthy females. Neurosci Lett, 338:123-6.
Tsang H, Lam P, Ng B, et al. 2000. Predictors of employment outcome for people with psychiatric disabilities: A review of the literature since the mid '80s. J Rehabil, 66:19-31.

Tulsky D, Zhu J, Ledbetter MF. 1997. WAIS-III WMS-III technical manual. San Antonia, TX: Psychological Corporation.

Wechsler D. 1997. The Wechsler Adult Intelligence Scale-third edition. San Antonio, TX: The Psychological Corporation.

Xu K, Liu XH, Nagarajan S, et al. 2002. Relationship of the delta-opioid receptor gene to heroin abuse in a large Chinese case/control sample. Am J Med Genet, 110:45-50. 